\title{
Use of histopathology services by general practitioners: recent changes in referral practice
}

\author{
K Shorrock
}

\begin{abstract}
Aims-To determine the nature and magnitude of the histopathological workload generated by specimens received from general practitioners and to assess the trends in referral practice.

Methods-All material submitted by general practitioners to the Leicester district histopathology service from 1989 to January 1993 was identified from departmental records. All GP referrals from October to December 1992 were also analysed. Total numbers of referrals from all sources were used for comparison. Specimens were also analysed according to diagnostic categories.

Results-There has been a progressive rise, both in the absolute number and the proportion of specimens relative to other surgical specimens submitted by GPs. Most are skin biopsy specimens. There were clear changes over the study period in the relative proportion of different types of lesions received, with a substantial increase in samples of benign naevi and papillomas. There was some evidence of a corresponding decrease in the number of these lesions submitted by hospital practitioners. The number of malignant skin tumours from GPs was small and the proportion had not increased over the study period.

Conclusions-Histopathological workload generated by GPs is increasing but it still represents a small proportion of the total. The major increase is in benign skin lesions.
\end{abstract}

(F Clin Pathol 1993;46:989-992)

As a result of the national waiting list initiative general practitioners are being encouraged to perform more minor surgery. It has been suggested that a number of benefits will result. These include a reduction in the number of referrals to hospital, particularly to dermatology, general surgery, and rheumatology clinics. Patients will further benefit from: a reduction in waiting time, avoidance of the need to travel; and treatment in a familiar environment. A pilot study has shown that the benefits are appreciated by patients. ${ }^{1}$ Financial savings to the NHS are also antici- pated, ${ }^{2}$ although it has been suggested that costs will increase. ${ }^{3}$

Minor surgery in general practice is expected to expand rapidly. This is likely to have major implications for the provision of histopathology services. The effects are already being felt and recent studies have shown a significant increase in the number of specimens submitted by GPs for histopathological examination. ${ }^{4-6}$

The full employment and revenue consequences of this initiative can only be assessed if accurate and complete data are available. This study analyses the trends in referral of material for histopathological interpretation by general practitioners in a large, single district service with a clearly defined catchment area.

\section{Methods}

The period since January 1989 was selected for study. This includes the time when the new GP contracts became operational. All specimens received from general practitioners for histopathological examination in the four month periods October to January every year since October 1989 were identified from departmental records. The total was compared with the total surgical workload of the department and the percentage of specimens derived from GPS was calculated.

January of each year was selected as an index month and a detailed breakdown of the GP specimens received in this month from 1989 to 1993 was performed. This month was selected because examination of laboratory activity showed variation between different years which was proportional to overall activity throughout the year. Random sampling proved difficult and the results showed comparable trends with those from analysis of single months. The specimens from general practitioners were subdivided according to site and diagnostic category. The total number of skin biopsy specimens received from all sources during these months was also established, as was the number of intradermal naevi. These data provided information regarding temporal trends in referral practice. All specimens received in October, November, and December 1992 were similarly analysed. The data from the period from October 1992 to January 1993 were used to 


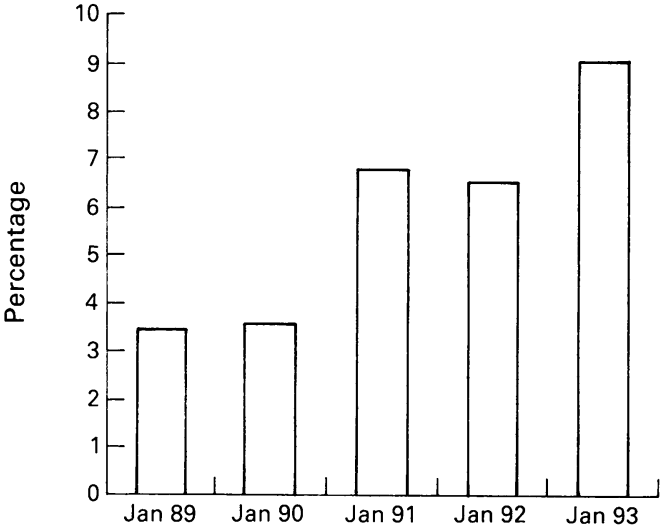

Figure 1 Specimens from GPs as a proportion of total.

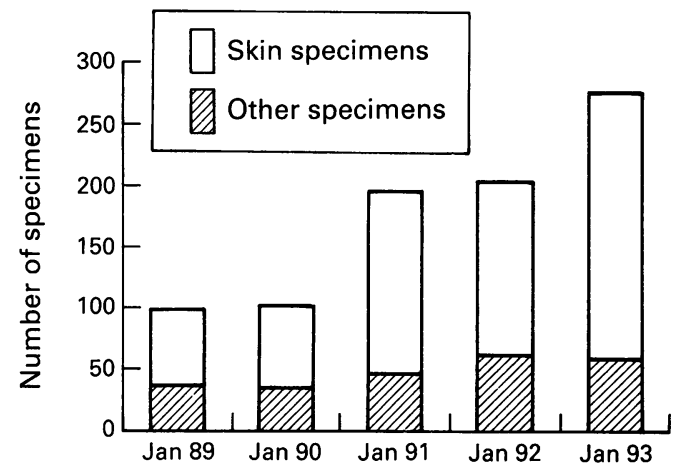

Figure 2 GP biopsy specimens taken according to site.

establish the commonest categories of lesion currently submitted.

Specimens from GPs were divided into skin biopsy specimens and others. A detailed analysis of the skin biopsy specimens was performed according to pathological diagnosis. The most commonly occurring lesions and all malignant skin tumours were selected for further study to establish the temporal trends in referral rate. No further analysis was performed on the non-dermatological specimens.

In this study no attempt was made to correlate actual diagnosis with clinical diagnosis or to assess adequacy of excision or quality of clinical information provided on request forms.

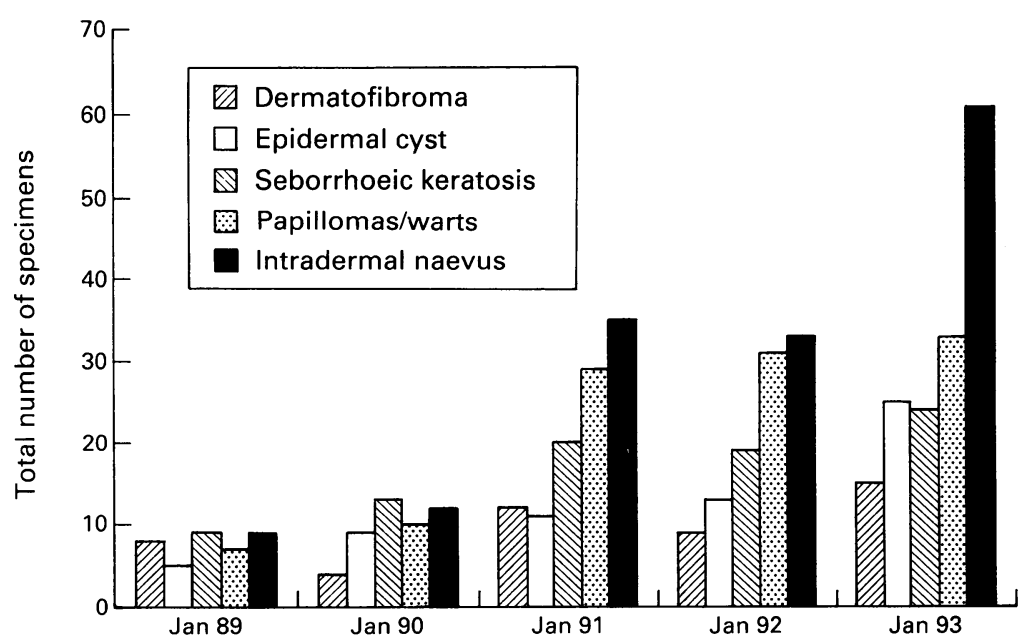

Figure 3 Numbers of lesions most commonly submitted by GPs.
Table 1 Most common skin lesions excised by GPs (October 1992 to fanuary 1993)

Total skin specimens (GP)

Intradermal naevus

Seborrhoeic keratosis

Epidermal cyst

Fibroepithelial polyp

Dermatofibroma

Viral wart

Inflammation/hyperplasia

Squamous papilloma

Basal cell carcinoma

Haemangioma

Actinic keratosis

Pyogenic granuloma

\section{Results}

The number of specimens received from GPs in the four year period has increased progressively and it now constitutes about $9 \%$ of our total caseload (fig 1). Skin biopsy specimens constitute the majority of specimens and the proportion of these shows the most substantial increase (fig 2). There has been a small increase in the number of specimens submitted for histological or cytological examination from other sites, but there has been no significant change in the referral pattern of these during this period.

A wide range of skin lesions are submitted by GPs. Those most commonly seen in the period October 1992 to January 1993 are listed in table 1. During the past five years there has been a noticeable change in the proportions of material received (fig 3). The absolute number of all lesions has increased, but this is most obvious in the case of intradermal naevi and benign papillomas and warts. Until the last month of study the increasing number of intradermal naevi

Table 2 Malignant and potentially premalignant skin lesions excised and biopsied by GPs (October 1992 to fanuary 1993)

\begin{tabular}{lr}
\hline Total skin specimens (GPs) & 871 \\
Malignant: & 27 \\
Basal cell carcinoma & 5 \\
Squamous carcinoma & 3 \\
Malignant melanoma & 3 \\
Metastatic/other & 17 \\
Preinvasive: & 7 \\
Actinic keratosis & 7 \\
Bowen's disease & \\
Keratoacanthoma & \\
\hline
\end{tabular}

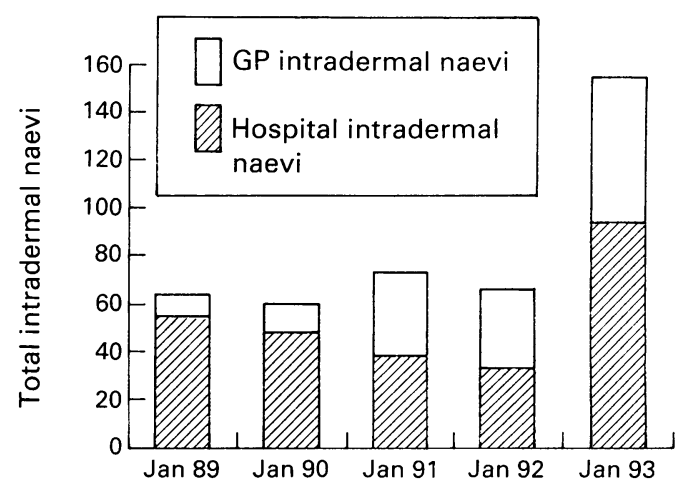

Figure 4 Relative proportions of intradermal naevi received from GPs and hospitals. 


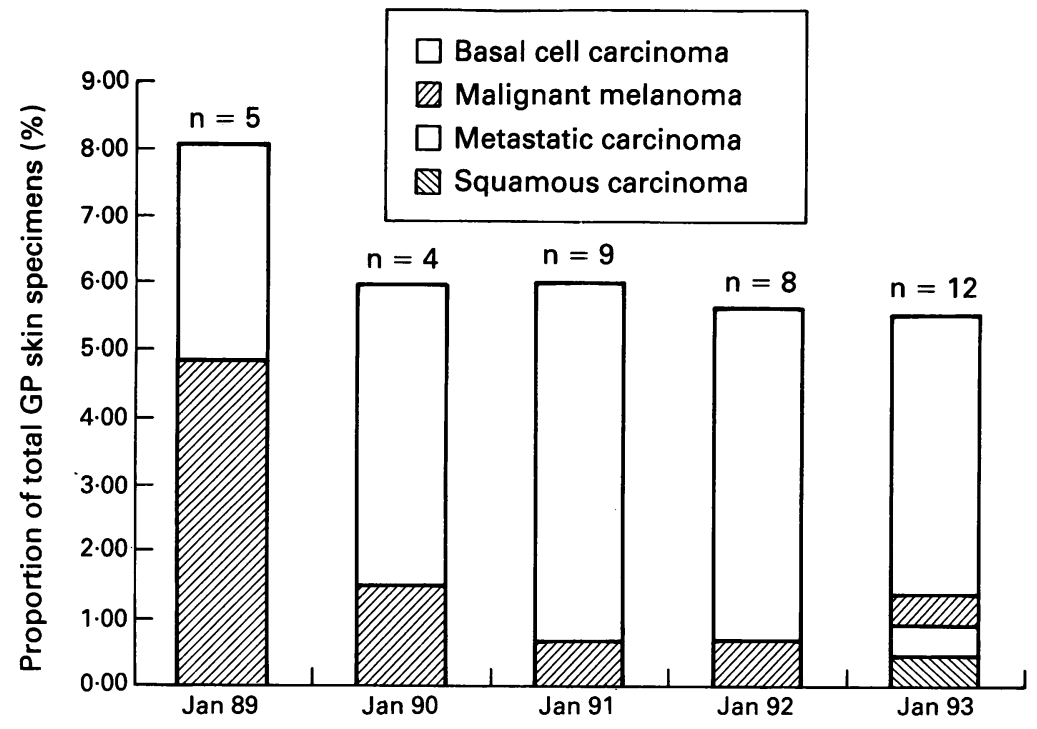

Figure 5 Malignant skin lesions submitted by GPs.

received from GPs corresponded to a decrease in those received from hospital practitioners. A recent local waiting list initiative, whereby additional sessions for dermatological minor surgery have been funded, has distorted the figures for January 1993 (fig 4).

Few malignant lesions are submitted by general practitioners (table 2). Most are basal cell carcinomas. We also receive a small number of squamous carcinomas and the occasional malignant melanoma. Neither the absolute number of these nor the proportion of malignant tumours to benign lesions has increased (fig 5). A significant number of biopsy specimens of preinvasive lesions; Bowen's disease and actinic keratoses are also submitted (table 2).

\section{Discussion}

Accurate and useful data concerning the effect of the increase in minor surgery in general practice on provision of histopathology services are difficult to collect. Frequently multiple laboratories serve overlapping populations. Therefore knowledge of referral habits by local practitioners is required before conclusions can be drawn. Leicester is a large, single district service which serves a clearly defined area. It is therefore suited to a study of this type. Many authors have attempted to address matters such as adequacy of excision of lesions, method of fixation, and quality of clinical information provided by the referring practitioner. ${ }^{56}$ These are difficult to assess retrospectively and to quantitate. Prospective studies are therefore required to evaluate them adequately. In the present series these issues have not been explored in detail.

These results confirm the pronounced recent increase in the number of skin biopsy specimens received from general practitioners. The threefold increase over the past four years is similar to that noted in previous studies.4-6 This clearly reflects a corresponding increase in the volume of minor surgery that is being performed by GPs. It is unlikely to represent the full picture, however, because the numbers of specimens, which are discarded without undergoing histopathological examination, are not known. Despite a widely, but not uniformly, held opinion that all excised specimens should be examined, ${ }^{6-8}$ there is abundant anecdotal evidence that this does not invariably occur.

The increase in volume of material received has not been evenly distributed and we have experienced a disproportionate increase in the number of benign naevi, papillomas, and warts. This could be interpreted as indicating a recent change in the criteria for recommending excision rather than other non-surgical treatment. There is a widespread, albeit unsubstantiated, belief that this is occurring. If this is the case, continued audit may reveal a progressive fall in the referral rate of these lesions as the number still available for excision diminishes. Alternatively, the increase may indicate a trend towards excision of these lesions by GPs rather than referral to a hospital clinic for excision. It was anticipated that this would occur. Our data concerning intradermal naevi (fig 4) suggest that this is indeed the case, although the results are inevitably obscured by other changes in hospital practice that have been stimulated by the waiting list initiative.

There is a small, but increasing, number of skin biopsy specimens from GPs that show non-specific inflammation or epidermal hyperplasia. Biopsy in these cases does not ultimately benefit the patient by providing a definite diagnosis. We also receive a substantial number of biopsy specimens from foci of Bowen's disease (squamous carcinoma in situ). This diagnosis does not usually seem to have been considered by the referring doctor. Dermatological referral is preferable to biopsy if there is clinical doubt as to the nature of the abnormality, particularly in the case of rashes. ${ }^{4}$ Although the clinical differential diagnosis is rarely stated on the referral forms, our experience supports this point of view.

There has been widespread concern that the minor surgery initiative would lead to a significant increase in the number of malignant lesions being misdiagnosed and inappropriately treated. At least two studies have identified a recent significant increase in the proportion of these submitted by GPs. ${ }^{89} \mathrm{We}$ have received few malignant lesions from this source and the proportion in our district is not increasing. Most malignant tumours in our experience have been basal cell and squamous carcinomas, and in these cases further active treatment is probably not required after adequate local excision. Occasional keratoacanthomas are submitted by GPs and these can be difficult to differentiate histologically from squamous carcinoma, particularly if inadequate clinical information is provided or if the lesion extends to the excision margins. Detailed analysis of the small number of malignant melanomas in this series is clearly 
impossible. A proportion of benign naevi in our experience are submitted with a history of recent increase in size or other clinically suspicious features and the possibility of malignancy is raised by the referring practitioner. It is argued that primary investigation and subsequent management of potentially malignant melanocytic lesions should be undertaken in departments that have experience in this field. Alternatively, general practitioners on the minor surgical list should undertake training in the management of these. ${ }^{9}$ Our experience is that the few malignant melanomas received by us from GPs are promptly and appropriately referred for definitive treatment when the diagnosis has been made histologically.

Specimens submitted by general practitioners still constitute a small, but progressively increasing, proportion of our total surgical workload, but this may well lead ultimately to a reduction in the rate of referral from hospital sources. Most of these biopsy specimens are diagnostically straightforward and occupy relatively little laboratory and medical staff time compared with other specimens. There is, however, a general awareness that further expansion of this activity will have revenue consequences for laboratories. ${ }^{110} \mathrm{~A}$ predicted catastrophic increase in laboratory workload has not occurred as yet. ${ }^{11}$ Our results do not support the contention that the GP minor surgery initiative will lead to a significant increase in the inappropriate excision of trivial lesions or of malignant tumours. ${ }^{3}$ This is a potential risk, however, particularly if performance targets are introduced. ${ }^{12}$ Continued audit will be required so that as GP minor surgery increases there are reliable data available on which future planning can be based. This has been highlighted as an area where early introduction of clinical audit is likely to be of major value. ${ }^{12}$

I thank Dr Alan Fletcher for his encouragement during the preparation of this study and for his helpful comments and criticisms of the paper.

1 Godfrey $\mathrm{E}$, Watkiss $\mathrm{M}$, Schneiden $\mathrm{H}$. Initiation and evaluation of a pilot $\mathrm{M}$, Schner for minor surgery in general ation of a pilot scheme for minor

2 Brown JS. Minor operations in general practice. $\mathrm{Br} \mathrm{Med} \mathcal{F}$ 1979;279:1609-10.

3 Shrank AB. Minor operations by General Practitioners. Lancet $1981 ; \mathrm{i}: 620$.

4 Cox NH, Wagstaff R, Popple AW. Using clinicopathological analysis of general practitioner skin surgery to determine educational requirements and guidelines. BMF 1992;304:93-6.

5 McWilliam LJ, Knox F, Wilkinson L, Oogarah P Performance of skin biopsies by general practitioners. PMF 1991;303:1177-9.

6 Hillan KJ, Johnson CP, Morton R. Effect of general practitioner contract on referral of specimens for histological titioner contract on referral of specim

7 Slater D. Performance of skin biopsies by general practitioners. $B M \mathcal{F}$ 1991;303:1472.

8 Williams RB, Burdge AH, Lewis Jones S. Skin biopsy in general practice. BMF 1991;303:1179-80.

9 Herd RM, Hunter JAA, McLaren KM, Chetty U, Watson ACH, Gollock JM. Excision biopsy of malignant melanoma by general practitioners in south east Scotland 1982-91. BMF 1992;305:1476-8.

10 Galloway M, Senadhira A. Labs take the strain. Health Service fournal 1990;100:1037.

11 Slater D. Screening and the 1990 contract. BMf 1990; 300:1074.

12 Milne $\mathrm{R}$. Minor surgery in general practice. $\mathrm{Br} \mathcal{F}$ Gen Pract 1990;40:175-6. 